\title{
Acknowledgement to Reviewers of Actuators in 2015
}

\author{
Actuators Editorial Office \\ Published: 22 January 2016 \\ MDPI AG, Klybeckstrasse 64, CH-4057 Basel, Switzerland; actuators@mdpi.com
}

The editors of Actuators would like to express their sincere gratitude to the following reviewers for assessing manuscripts in 2015.

We greatly appreciate the contribution of expert reviewers, which is crucial to the journal's editorial decision-making process. Several steps have been taken in 2015 to thank and acknowledge reviewers. Good, timely reviews are rewarded with a discount off their next MDPI publication. By creating an account on the submission system, reviewers can access details of their past reviews, see the comments of other reviewers, and download a letter of acknowledgement for their records. We are grateful to our reviewers for the contribution they make.

$\begin{array}{lll}\text { Abramovich, Haim } & \text { Koganezawa, Koichi } & \text { Rudykh, Stephan } \\ \text { Aktakka, Ethem } & \text { Kottapalli, Ajay } & \text { Seung-Bok, Choi } \\ \text { Alonso, J Marcos } & \text { Kuckling, Dirk } & \text { Shafik, Mahmoud } \\ \text { Aoyagi, Takahiro } & \text { La, H.m. } & \text { Shang, Weiwei } \\ \text { Aphale, Sumeet S. } & \text { Lee, Jae-shin } & \text { Song, Fei } \\ \text { Asbeck, Alan } & \text { Lexcellent, Christian } & \text { Tjahjowidodo, Tegoeh } \\ \text { Aw, K.C. } & \text { Li, Qiang } & \text { Vos, Roelof } \\ \text { Bilgen, Onur } & \text { Ling, Jiazhen } & \text { Wallaschek, Jörg } \\ \text { Bo, Cheng } & \text { Lofland, Jr., Samuel E. } & \text { Wang, Yongquan } \\ \text { Cagliani, Alberto } & \text { Lopez, Javier } & \text { Webber, Kyle } \\ \text { Casals, Alícia } & \text { Lu, Yang } & \text { Wen, Weijia } \\ \text { Chen, Silu } & \text { Lumia, Ron } & \text { Wen, Chih-Yung } \\ \text { Chng, Choon-Peng } & \text { Mathieson, Andrew } & \text { Wereley, Norman M. } \\ \text { Cianchetti, Matteo } & \text { Merola, Alessio } & \text { Woods, Ben } \\ \text { Cuculovic, Milos } & \text { Nakano, Kimihiko } & \text { Wu, Jiagang } \\ \text { Dobson, Jon } & \text { Oh, Il Kwon } & \text { Wu, Chyan-Chyi } \\ \text { Dong, Shuxiang } & \text { Orlando, Calogero } & \text { Wu, Yinghao } \\ \text { Feng, Yue } & \text { Palacin, Jordi } & \text { Yeatman, Eric } \\ \text { Feng, Zhi Hua } & \text { Palli, Gianluca } & \text { Yoshimura, T. } \\ \text { Gapanov, Igor } & \text { Park, Hoon Cheol } & \text { Zhang, Jianzhong } \\ \text { Gei, Massimiliano } & \text { Pérez, Jacinto Cortéz } & \text { Zhao, Hongwei } \\ \text { Giunta, Gaetano } & \text { Philen, Michael } & \text { Zhao, Ya-Pu } \\ \text { Guarnieri, Daniela } & \text { Plante, Jean-Sébastien } & \text { Zheng, Jinchuan } \\ \text { Hu, Zhongxu } & \text { Qiu, Zhicheng } & \text { Zhou, Miao-lei } \\ \text { Huang, Zhaorong } & \text { Rabindran, Dinesh } & \text { Zhu, Limin } \\ \text { Karaca, Haluk E. } & \text { Rocheleau, David } & \text { Zhu, Benpeng } \\ \text { Kim, } & & \end{array}$

Kim, I.S. 